\title{
Hábitos alimenticios, nocivos y rendimiento académico en estudiantes universitarios en tiempos de Covid-19
}

\author{
Eating habits, harmful and academic performance in university students in times of Covid-19
}

Hábitos alimentares, desempenho prejudicial e acadêmico em estudantes universitários em tempos de Covid-19

\section{Sara Elizabeth Bravo Salinas}

sara.bravo@ucacue.edu.ec https://orcid.org/0000-0003-4878-1662

\section{Diana Carolina Izquierdo Coronel}

dizquierdoc@ucacue.edu.ec https://orcid.org/0000-0002-1577-491X

\author{
Alex Alberto Castillo Zhizhpón \\ acz8@hotmail.com \\ https://orcid.org/0000-0002-6487-7552 \\ Paola Andrea Rodas Bermeo \\ paolarodas18@hotmail.com \\ https://orcid.org/0000-0003-2834-7535
}

\section{Universidad Católica de Cuenca, Azogues-Ecuador}

Recibido 4 de octubre 2021 / Arbitrado y aceptado 12 de noviembre 2021 / Publicado 30 de diciembre 2021

\begin{abstract}
RESUMEN
Los hábitos alimenticios influyen drásticamente en el rendimiento académico universitario, teniendo consecuencias a corto, mediano y largo plazo. Objetivo. Identificar los hábitos alimenticios nocivos y rendimiento académico en estudiantes universitarios en tiempos de Covid-19 en Ecuador-Azogues. Metodología. Estudio descriptivo transversal, población de estudio 623 estudiantes universitarios de la carrera de Medicina, se les aplicó una encuesta, el análisis estadístico se lo realizó mediante SPSS V20. Resultados. El 64,7\% corresponden al sexo femenino, 58,3\% proceden de la provincia del Cañar, el 82,7 son católicos y el $10,1 \%$ presentan bajo rendimiento, de los cuales el $28,6 \%$ presentaron sobrepeso y la probabilidad de que estos estudiantes tengan bajo rendimiento fue del $63 \%$. Se demostró también que el $82,5 \%$ de estudiantes consumen comida chatarra, existiendo 3 veces (RP 3,12; IC95\% 1,88-5,26) más probabilidad de que éstos estudiantes tengan bajo rendimiento, el sexo masculino también presentó 1,6 veces (IC95\% 1,04-2,65) más probabilidad de tener bajo rendimiento en comparación con el sexo femenino, mientras que los estudiantes que proceden de las distintas provincias tienen 1,6 veces (RP 1,63; IC95\% 1,03-2,63) más probabilidad de tener rendimiento deficiente. Un 85,7\% de estudiantes consume alcohol y al menos el $25,4 \%$ ha consumido drogas existiendo 2 veces (PR 2; IC95\% 1,19-3,44) más probabilidad de que estos estudiantes bajen su rendimiento. Conclusiones. Se determinó que el bajo rendimiento académico está relacionado no solo por el sexo sino también, por la procedencia, la malnutrición, consumo de comida chatarra y uso de drogas incrementando la proporción de estudiantes con bajo rendimiento.
\end{abstract}

Palabras clave: Covid-19; Hábitos alimenticios; Rendimiento académico; Universitarios

\begin{abstract}
Eating habits drastically influence university academic performance, having short, medium and long term consequences. Objective. To identify harmful eating habits and academic performance in university students in times of Covid-19 in Ecuador-Azogues. Methodology. Descriptive cross-sectional study, study population 623 university students of the Medicine career, a survey was applied to them, the statistical analysis was carried out using SPSS V20. Results. $64.7 \%$ were female, $58.3 \%$ were from the province of Cañar, $82.7 \%$ were Catholic and $10.1 \%$ were underachievers, of which $28.6 \%$ were overweight and the probability of these students being underachievers was $63 \%$. It was also shown that $82.5 \%$ of students consume junk food, with 3 times (PR $3.12 ; 95 \% \mathrm{Cl} 1.88-5.26)$ more probability that these students have low performance, the male sex also presented 1.6 times $(95 \% \mathrm{Cl} 1.04-2.65)$ more probability of having low performance compared to the female sex, while students coming from the different provinces have 1.6 times $(\mathrm{PR} 1.63 ; 95 \% \mathrm{Cl} 1.03-2.63)$ more probability of having poor performance. A $85.7 \%$ of students consume alcohol and at least $25.4 \%$ have consumed drugs existing 2 times (PR 2; 95\% Cl 1.19-3.44) more likely that these students have lower performance. Conclusions. It was determined that low academic performance is related not only by gender but also by origin, malnutrition, consumption of junk food and drug use, increasing the proportion of students with low performance.
\end{abstract}

Key words: Covid-19; Eating habits; Academic performance; University students 


\section{RESUMO}

Os hábitos alimentares influenciam drasticamente o desempenho acadêmico universitário, tendo conseqüências a curto, médio e longo prazo. Objetivo. Identificar hábitos alimentares prejudiciais e desempenho acadêmico em estudantes universitários durante o período Covid-19 no Equador-Azogues. Metodologia. Estudo descritivo transversal, população estudada 623 estudantes universitários de Medicina, uma pesquisa foi aplicada a eles, a análise estatística foi realizada utilizando o SPSS V20. Resultados. $64,7 \%$ eram do sexo feminino, $58,3 \%$ eram da província de Cañar, $82,7 \%$ eram católicos e $10,1 \%$ eram alunos com baixo aproveitamento, dos quais $28,6 \%$ tinham excesso de peso, e a probabilidade de esses alunos serem alunos com baixo aproveitamento era de $63 \%$. Também foi demonstrado que $82,5 \%$ dos estudantes consomem comida de plástico, com 3 vezes (PR $3,12$; $95 \% \mathrm{Cl} 1,88-5,26)$ mais propensos a serem sub-atingidos, os estudantes do sexo masculino também foram 1,6 vezes $(95 \% \mathrm{Cl}$ 1,04-2,65) mais propensos a serem sub-atingidos em comparação com os estudantes do sexo feminino, enquanto os estudantes das diferentes províncias foram 1,6 vezes ( $P R \quad 1,63 ; 95 \% \mathrm{Cl} 1,03-2,63$ ) mais propensos a serem sub-atingidos. $85,7 \%$ dos estudantes usam álcool e pelo menos $25,4 \%$ usaram drogas sendo 2 vezes (PR 2; $95 \% \mathrm{Cl} 1,19-3,44)$ mais propensos a ter um desempenho mais fraco. Conclusões. Constatou-se que o baixo desempenho acadêmico estava relacionado não apenas por gênero, mas também, por formação, desnutrição, consumo de junk food e uso de drogas, aumentando a proporção de estudantes com baixo desempenho

Palavras-chave: Covid-19; Hábitos alimentares; Desempenho acadêmico; Estudantes universitários

\section{INTRODUCCIÓN}

La presencia de SARS COV2 se convirtió en un problema de salud pública a nivel mundial, con una taza de letalidad promedio de 3-5\% en varios países, sin embargo, Perú y México supera el $9 \%$, en la mayoría de los casos su fácil y rápida propagación de contagios ha llegado a saturar y colapsar los sistemas de salud (pública y privada) $(1,2)$, por tal razón, varios países tomaron medidas de prevención como el confinamiento social, la limitada capacidad de movilizarse dio como resultado cambios en el estilo de vida de las personas, el sedentarismo, cambios en los hábitos alimenticios, alteraciones del sueño y hábitos nocivos son algunas de las modificaciones que las personas presentaron, sea por el acceso a los alimentos, por motivos económicos, emocionales o físicos (3).

En varias investigaciones durante la última década se ha determinado que entre el $50-60 \%$ de estudiantes de carreras relacionadas con salud, bienestar y nutrición tienen adecuados conocimientos sobre sobre hábitos de vida saludable, sin embargo, a penas entre $15-20 \%$ de éstos estudiantes ponen en práctica dichos hábitos (4), ya que la mayoría de la población universitaria opta por consumir comida rápida (chatarra) con altos niveles de grasas saturadas, bebidas procesadas gasificadas y energizadas, atribuyendo dichos hábitos inadecuados a la gran carga horaria académica y falta de tiempo, esto a su vez, ha demostrado estar relacionado con un bajo rendimiento académico lo que se ha evidenciado a nivel mundial (4-8).

El rendimiento académico implica para los docentes y estudiantes la necesidad de cumplir con una serie de metas y objetivos previamente establecidos, los cuales quedan registrados por un sistema de calificación producto de una evaluación, la misma determinará o no la superación de los distintos niveles de educación en el sistema actual. El estado nutricional de los estudiantes es un factor que influye en el rendimiento académico y el aprendizaje, la mal nutrición afecta dicho aprendizaje, al existir una alteración de las funciones cognitivas, así como también una mayor predisposición a enfermar, aumentando de esta manera el ausentismo, el fracaso escolar y aparición de enfermedades crónicas metabólicas no transmisibles a mediano y largo plazo (9). 
La actual pandemia con el aislamiento social y el confinamiento, han afectado de manera significativa no solo en la salud mental de las personas, sino también en la aparición de enfermedades crónicas metabólicas con consecuencias que amenazan la aparición de estas enfermedades (10). Estas alteraciones pueden traducirse en aislamiento emocional, confusión, estigma, que desencadena una variedad de condiciones emocionales como (angustia, condiciones psiquiátricas, hábitos poco saludables, tendencia al consumo de sustancias nocivas), adquisición de malos hábitos alimenticios y nocivos (11).

Por ello la importancia de realizar esta investigación para determinar los principales hábitos alimenticios nocivos adquiridos y ejecutados durante el confinamiento como consecuencia de la pandemia y a su vez, como influyeron éstos hábitos en el rendimiento académico de nuestros estudiantes.

\section{MATERIALES Y MÉTODOS}

Estudio descriptivo de corte transversal de caracterización de hábitos nutricionales nocivos y rendimiento académico en estudiantes universitarios de Medicina. Para la caracterización, se realizó estadística descriptiva, con cálculos de Media y Desviación Estándar. Posterior a la caracterización, se establecen correlaciones entre: a)
Antropometría y Rendimiento académico, b) Hábitos alimenticios, nocivos y rendimiento académico, usando el coeficiente de Pearson $(p<0,05)$ y el Coeficiente de Tau - Kendall $(p<0,05)$.

El instrumento utilizado para la recolección de datos fue una encuesta que consta de varios bloques: Bloque sociodemográfico, salud sexual y reproductiva, familiar, nutricional - hábitos y psicológico; mismos que fueron revisados y avalados por la Jefatura de Investigación e Innovación de la Universidad Católica de Cuenca al declarar al Proyecto “Factores asociados al rendimiento académico en estudiantes universitarios en tiempos de Covid-19" como ganador en la convocatoria denominada "Proyectos de Investigación COVID-19" generada a raíz de la emergencia sanitaria en el 2020, por lo tanto, esta investigación deriva de dicho proyecto.

La población de estudio estuvo constituida por los estudiantes de la carrera de medicina de la Universidad Católica de Cuenca sede Azogues, que se matricularon legalmente en el periodo académico octubre 2020 marzo 2021 a partir del segundo ciclo académico hasta el décimo ciclo y aceptaron participar de forma voluntaria, sin ejercer procedimientos de coacción ni coerción. De los 1600 estudiantes matriculados durante el periodo académico mencionado, 623 estudiantes decidieron participar en el estudio. 


\section{RESULTADOS Y DISCUSIÓN}

En el estudio participaron 623 estudiantes de la Facultas de Salud y bienestar en la carrera de Medicina de la Universidad Católica de Cuenca sede Azogues, con edades comprendidas entre 18 y 36 años, la edad promedio fue de 20,8 años $( \pm 0,8)$, con una mediana de 20 años.
El $64,7 \%$ de la muestra $\left(\mathrm{IC}_{95 \%} 60,19-67,73\right)$ corresponden al sexo femenino, 363 (58,3\%; $\left.\mathrm{IC}_{95 \%} 54,09-61,79\right)$ proceden de la provincia de Cañar, 515 (82,7\%; IC $C_{95 \%}$ 78,97-84,91) manifestaron ser católicos, 597 (95.8\% IC $\mathrm{IS}_{95}$ 93,25-96,62) participantes son solteros, los datos se muestran en la Tabla 1.

Tabla 1. Datos socio-demográficos de los participantes.

\begin{tabular}{|c|c|c|c|c|}
\hline Variable & Categoría & $\mathbf{N}$ & $\%$ & $I_{95 \%}$ \\
\hline \multirow[t]{3}{*}{ Sexo } & Hombre & 220 & 35,3 & $31,30-38,84$ \\
\hline & Mujer & 403 & 64,7 & $60,19-67,73$ \\
\hline & Total & 623 & 100,0 & \\
\hline \multirow[t]{3}{*}{ Procedencia } & Cañar & 363 & 58,3 & $54.09-61.79$ \\
\hline & Otras provincias & 260 & 41,7 & $37.07-44.94$ \\
\hline & Total & 623 & 100,0 & \\
\hline \multirow[t]{7}{*}{ Religión } & Católica & 515 & 82,7 & $78,97-84,91$ \\
\hline & Evangélica & 10 & 1,6 & $0,32-1,92$ \\
\hline & Cristiana & 44 & 1,6 & $0,32-1,92$ \\
\hline & Testigos de Jehová & 4 & 7,1 & $5,13-8,98$ \\
\hline & Ateo & 10 & 0,6 & $4,17-786$ \\
\hline & Otros & 40 & 6,4 & $4,49-8,34$ \\
\hline & Total & 623 & 100,0 & \\
\hline \multirow[t]{6}{*}{ Estado Civil } & Solteros & 597 & 95,8 & $93,25-96,62$ \\
\hline & Casados & 14 & 2,2 & $0,96-3,21$ \\
\hline & Divorciados & 3 & 0,5 & $0,16-1,12$ \\
\hline & Unión libre & 9 & 1,4 & $0,48-2,40$ \\
\hline & Separados & 0 & 0,0 & \\
\hline & Total & 623 & 100,0 & \\
\hline
\end{tabular}

$\mathrm{IC}_{95 \%}$ : Intervalo de Confianza del 95\%

Fuente: Estudio “Factores asociados al rendimiento académico en estudiantes universitarios en tiempos de Covid-19” 
En cuanto al rendimiento académico, en la Tabla 2 se refleja que del total de participantes 63 $\left(10,1 \% I_{95 \%} 7,70-12,35\right)$ reflejaron mal rendimiento de éstos $36\left(57,1 \% I_{95 \%} 44,44-69,84\right)$ reprobaron el ciclo académico.

Tabla 2. Datos del rendimiento académico de los participantes.

\begin{tabular}{llccc}
\hline \multicolumn{1}{c}{ Variable } & Categoría & $\mathbf{N}$ & $\%$ & IC $_{\mathbf{9 5} \%}$ \\
\hline Rendimiento académico & Categoría & N & $\%$ & $29,37-36,75$ \\
& Sobresaliente & 209 & 33,5 & $52,16-59,87$ \\
& Bueno - notable & $\mathbf{3 5 1}$ & $\mathbf{5 6 , 3}$ & $7,70-12,35$ \\
& Regular - Malo & 63 & 10,1 & \\
\multirow{3}{*}{ Reprobados } & Total & 477 & 100,0 & $44,44-69,84$ \\
& Si & $\mathbf{3 6}$ & $\mathbf{5 7 , 1}$ & $20,15-53,96$ \\
& No & 27 & 42,9 & \\
& Total & $\mathbf{6 3}$ & $\mathbf{1 0 0 , 0}$ & \\
\hline
\end{tabular}

$\mathrm{IC}_{95 \%}$ : Intervalo de Confianza del $95 \%$

Fuente: Estudio "Factores asociados al rendimiento académico en estudiantes universitarios en tiempos de Covid-19"

En cuanto al estado nutricional y $18\left(28,6 \% \quad \mathrm{IC}_{95 \%} \quad 17,46-39,68\right)$ se encontraron percepción de hábitos de los estudiantes con con sobrepeso, de estos el 77,8\% reconocen bajo rendimiento como se muestra en la Tabla que sus hábitos alimenticios no son los más 3, 31 participantes $\left(49,2 \% \quad I_{95 \%} 36,50-61,90\right)$ adecuados, considerando que podrían mejorar presentaron normopeso, sin embargo, de los dichos hábitos.

estudiantes que presentaron mal nutrición

Tabla 3. Estado nutricional y percepción de hábitos.

\begin{tabular}{|c|c|c|c|c|c|}
\hline & Variable & Categoría & $\mathbf{N}$ & $\%$ & $I_{95 \%}$ \\
\hline \multirow[t]{5}{*}{ IMC } & & Bajo peso & 7 & 11,1 & $3,17-19,04$ \\
\hline & & Normopeso & 31 & 49,2 & $36,50-61,90$ \\
\hline & & Sobrepeso & 18 & 28,6 & $17,46-39,68$ \\
\hline & & Obesidad & 7 & 11,1 & $3,17-19,04$ \\
\hline & & Total & 63 & 100,0 & \\
\hline
\end{tabular}




\begin{tabular}{llccc}
\hline \multicolumn{1}{c}{ Variable } & Categoría & N & \% & IC $_{\mathbf{9 5 \%}}$ \\
\hline Percepción sobre sus hábitos & Buenos & 3 & 16,6 & $5,56-33,33$ \\
alimenticios & Excelentes & 0 & 0 & \\
& Malos & $\mathbf{1}$ & $\mathbf{5 , 6}$ & $5,55-16,66$ \\
& Regulares & 14 & 77,8 & $55,56-94,44$ \\
& Total & 18 & 100,0 & \\
¿Crees que podrías mejorar tus & No & 4 & 22,2 & $5,56-44,44$ \\
hábitos alimenticios? & Si & 14 & 77,8 & $55,56-94,44$ \\
& Total & 18 & 100,0 & \\
\hline
\end{tabular}

$\mathrm{IC}_{95 \%}$ : Intervalo de Confianza del $95 \%$

Fuente: Estudio “Factores asociados al rendimiento académico en estudiantes universitarios en tiempos de Covid-19”

De los 63 participantes con bajo consumir bebidas gaseosas (colas) en este rendimiento $52\left(82,5 \% \quad I C_{95 \%} \quad 71,42-90,47\right)$ grupo en cambio, predominan los de sexo manifestaron consumir comida rápida (papas fritas, hamburguesas, hot dogs, nachos, entre otros) consumiendo al menos dos veces a la semana, de los cuales el $42,9 \%$ corresponde al sexo femenino; de igual forma un gran porcentaje $(66,7 \%)$ de estudiantes refirieron masculino con el 38,1\%, los datos se evidencian en la Tabla 4, así también se demuestra que solo $18\left(28,6 \% \quad I C_{95 \%}\right.$ 17,46-39,68) estudiantes manifestaron consumir bebidas energizantes de estos la mayor proporción se encuentra en el sexo femenino.

Tabla 4. Consumo de comida chatarra y bebidas azucaradas.

\begin{tabular}{|c|c|c|c|c|c|c|c|c|}
\hline \multirow{3}{*}{ Variable } & \multirow{3}{*}{ Categoría } & \multicolumn{4}{|c|}{ SEXO } & \multirow{3}{*}{ Total } & \multirow{3}{*}{$\%$} & \multirow{3}{*}{ IC $_{95 \%}$} \\
\hline & & \multicolumn{2}{|c|}{ Hombres } & \multicolumn{2}{|c|}{ Mujeres } & & & \\
\hline & & $\mathbf{N}$ & $\%$ & $\mathbf{N}$ & $\%$ & & & \\
\hline \multirow{3}{*}{$\begin{array}{l}\text { Consumo de comida } \\
\text { rápida }\end{array}$} & No & 5 & 7,9 & 6 & 9,5 & 11 & 17,5 & $7,93-26,98$ \\
\hline & $\mathrm{Si}$ & 25 & 39,7 & 27 & 42,9 & 52 & 82,5 & $71,42-90,47$ \\
\hline & Total & 30 & 47,6 & 33 & 52,4 & 63 & 100,0 & \\
\hline \multirow[t]{3}{*}{ Consumo de gaseosas } & No & 6 & 9,5 & 15 & 23,8 & 21 & 33,3 & $22,2-44,7$ \\
\hline & $\mathrm{Si}$ & 24 & 38,1 & 18 & 28,6 & 42 & 66,7 & $53,96-77,7$ \\
\hline & Total & 30 & 47,6 & 33 & 52,4 & 63 & 100,0 & \\
\hline \multirow{3}{*}{$\begin{array}{l}\text { Consumo de bebidas } \\
\text { energizantes }\end{array}$} & No & 17 & 27,0 & 28 & 44,5 & 45 & 71,4 & $60,31-82,53$ \\
\hline & $\mathrm{Si}$ & 13 & 20,6 & 5 & 7,9 & 18 & 28,6 & $17,46-39,68$ \\
\hline & Total & 30 & 47,6 & 33 & 52,4 & 63 & 100,0 & \\
\hline
\end{tabular}

$\mathrm{IC}_{95 \%}$ : Intervalo de Confianza del 95\%

Fuente: Estudio “Factores asociados al rendimiento académico en estudiantes universitarios en tiempos de Covid-19” 
Los resultados obtenidos en cuanto a los hábitos nocivos, actividad física y horas de sueño que se reflejan en la Tabla 5, evidencian que 53 (85,7\% IC I5\% $_{76,19-93,65)}$ de los 63 estudiantes consumen alcohol, con proporciones muy similares entre hombres y mujeres, sin embargo, el consumo de tabaco no supera el $16 \%$, existiendo un mayor predominio en el sexo masculino, en cambio el uso drogas supera el consumo de tabaco con un $25,4 \%\left(\mathrm{IC}_{95 \%}\right.$ 14,28-36,50), de la misma manera superado en el sexo masculino. Solo el $31,7 \%$ $\left(\right.$ IC $\left._{95 \%} 20,63-42,85\right)$, de los estudiantes realizan algún tipo de actividad física, y el 82,5\% (IC $71,42-90,47)$ manifestó tener menos de 7 horas de sueño diarias.

Tabla 5. Consumo de comida chatarra y bebidas azucaradas.

\begin{tabular}{|c|c|c|c|c|c|c|c|c|}
\hline \multirow{3}{*}{ Variable } & \multirow{3}{*}{ Categoría } & \multicolumn{4}{|c|}{ SEXO } & \multirow{3}{*}{ Total } & \multirow{3}{*}{$\%$} & \multirow{3}{*}{$I_{95 \%}$} \\
\hline & & \multicolumn{2}{|c|}{ Hombres } & \multicolumn{2}{|c|}{ Mujeres } & & & \\
\hline & & $\mathbf{N}$ & $\%$ & $\mathbf{N}$ & $\%$ & & & \\
\hline \multirow[t]{3}{*}{ Consumo de alcohol } & No & 4 & 6,4 & 5 & 7,9 & 9 & 14,3 & $6,34-23,80$ \\
\hline & $\mathrm{Si}$ & 26 & 41,3 & 28 & 44,4 & 54 & 85,7 & $76,19-93,65$ \\
\hline & Total & 30 & 47,7 & 33 & 52,3 & 63 & 100,0 & \\
\hline \multirow[t]{3}{*}{ Consumo de tabaco } & No & 22 & 34,9 & 31 & 49,2 & 53 & 84,1 & $74,60-92,06$ \\
\hline & $\mathrm{Si}$ & 8 & 12,7 & 2 & 3,2 & 10 & 15,9 & $7,93-25,39$ \\
\hline & Total & 30 & 47,6 & 33 & 52,4 & 63 & 100,0 & \\
\hline \multirow[t]{3}{*}{ Uso de drogas } & No & 18 & 28,6 & 29 & 46,1 & 47 & 74,6 & $63,49-84,12$ \\
\hline & $\mathrm{Si}$ & 12 & 19 & 4 & 6,3 & 16 & 25,4 & $14,28-36,50$ \\
\hline & Total & 30 & 47,6 & 33 & 52,4 & 63 & 100,0 & \\
\hline \multirow[t]{3}{*}{ Actividad física } & No & 7 & 11,1 & 13 & 20,6 & 20 & 31,7 & $20,63-42,85$ \\
\hline & $\mathrm{Si}$ & 23 & 36,6 & 20 & 31,7 & 43 & 68,3 & $55,5-79,36$ \\
\hline & Total & 30 & 47,7 & 33 & 52,3 & 63 & 100,0 & \\
\hline \multirow[t]{3}{*}{ Horas de sueño } & Más a 7H & 7 & 11,1 & 5 & 7,9 & 11 & 17,5 & $9,52-28,57$ \\
\hline & $\begin{array}{l}\text { Menos de } 7 \\
\text { horas }\end{array}$ & 23 & 36,6 & 28 & 44,4 & 52 & 82,5 & $71,42-90,47$ \\
\hline & Total & 30 & 47,7 & 33 & 52,3 & 63 & 100,0 & \\
\hline
\end{tabular}

IC $95 \%$ Intervalo de Confianza del $95 \%$

Fuente: Estudio “Factores asociados al rendimiento académico en estudiantes universitarios en tiempos de Covid-19”

La correlación de variables demostró que el sexo masculino tiene $66 \%\left(\operatorname{RP} 1,66 ; I_{95 \%}\right.$ 1,04-2,65) más probabilidad de tener bajo rendimiento que el sexo femenino, mientras que los estudiantes que proceden de las distintas provincias del país tienen el 63\%
(RP 1,63; IC I $_{95}$ 1,03-2,63) más probabilidad de tener bajo rendimiento, que aquellos que proceden de la provincia del Cañar (Tabla 6). En cambio, la religión no tiene significancia en los estudiantes para tener un bajo rendimiento (RP 1,14; IC $95 \%$ 0,38-1,17). 
Tabla 6. Características de los estudiantes con bajo rendimiento distribuidos por sexo, procedencia y religión.

\begin{tabular}{llrrrrrr}
\hline \multicolumn{1}{c}{ Variable } & \multicolumn{1}{c}{ Categoría } & N & \%a & RP & IC95\% & RV & Valor $\mathbf{p}$ \\
\hline Sexo & Hombre & 30 & 13,6 & 1,66 & $1,04-2,65$ & 1,70 & 0,03 \\
& Mujer & 33 & 8,2 & & & & \\
Procedencia & Cañar & 29 & 8,0 & 1,63 & $1,03-2,63$ & 1,75 & 0,04 \\
& Otras provincias & 34 & 13,1 & & & & \\
Religión & Católica & 48 & 9,3 & 1,49 & $0,85-2,56$ & 1,58 & 0,15 \\
& Otras religiones & 15 & 13,9 & & & & \\
\hline
\end{tabular}

c El denominador para el porcentaje corresponde a la variable de la fila

RP Razón de Prevalencia

IC95\% Intervalo de Confianza del 95\%

RV Razón de Verosimilitud

Fuente: Estudio “Factores asociados al rendimiento académico en estudiantes universitarios en tiempos de Covid-19”

EnlaTabla 7seevidenciaque los estudiantes que presentaban malnutrición tienen 1,6 veces (RP 1,$63 ; \quad I C_{95 \%} 1,02-2,63$ ) más probabilidad de tener bajo rendimiento académico que aquellos estudiantes que tienen normopeso, así mismo los que consumen comida rápida por lo menos dos veces a la semana tienen 3,2 veces (RP 3,12; IC $\mathrm{I}_{95}$ 1,88-5,26) más probabilidad de tener bajo rendimiento académico, en cuanto al consumo de bebidas gaseosas, energizantes, consumo de alcohol y tabaco no demostraron significancia alguna (RP 1,06; IC ${ }_{95 \%}$ 0,64-1,75),
(RP 1,23; IC $\left.{ }_{95 \%} 0,73-2,08\right),\left(R P 1,81 ; I C C_{95 \%} 0,90-\right.$ 3,57), (RP 1,06; IC $95 \%$ 0,56-2,04) respectivamente. Por el contrario, aquellos estudiantes que han consumido drogas tienen 2 veces (RP 2,81; $\left.\mathrm{IC}_{95 \%} 0,90-3,57\right)$ más probabilidad de tener bajo rendimiento que aquellos estudiantes que no la han consumido, la actividad física y las horas de sueño no demostraron ser significativos en el rendimiento académico de los estudiantes ya que el Coeficiente de Pearson y Tau de Kendall superaron el 0,05.

Tabla 7. Relación de los hábitos nutricionales, nocivos y físicos.

\begin{tabular}{llllllll}
\hline \multicolumn{1}{c}{ Variable } & \multicolumn{1}{c}{ Categoría } & N & \%a & RP & IC $_{\mathbf{9 5 \%}}$ & RV & Valor $\mathbf{p}$ \\
\hline IMC & Normopeso & 31 & 8,2 & 1,63 & $1,02-2,63$ & 1,72 & 0,04 \\
& Malnutrición & 32 & 13,2 & & & & \\
Comida rápida & Otras ocasiones & 27 & 7,2 & 3,12 & $1,88-5,26$ & 18,02 & $<0,05$ \\
& 2 veces a la semana & 25 & 22,3 & & & & \\
\hline
\end{tabular}




\begin{tabular}{|c|c|c|c|c|c|c|c|}
\hline Variable & Categoría & $\mathbf{N}$ & $\% a$ & $\mathbf{R P}$ & $I_{95 \%}$ & RV & Valor $\mathbf{p}$ \\
\hline \multirow[t]{2}{*}{ Gaseosas } & No consume & 21 & 9,7 & 1,06 & $0,64-1,75$ & 1,78 & 0,8 \\
\hline & Si consume & 42 & 10,3 & & & & \\
\hline \multirow[t]{2}{*}{ Bebidas energizantes } & No consume & 45 & 9,6 & 1,23 & $0,73-2,08$ & 1,69 & 0,4 \\
\hline & Si consume & 18 & 11,8 & & & & \\
\hline \multirow[t]{2}{*}{ Alcohol } & No consume & 9 & 6,3 & 1,81 & $0,90-3,57$ & 3,27 & 2,9 \\
\hline & Si consume & 54 & 11,2 & & & & \\
\hline \multirow[t]{2}{*}{ Tabaco } & No consume & 53 & 10 & 1,06 & $0,56-2,04$ & 0,03 & 0,8 \\
\hline & Si consume & 10 & 10,6 & & & & \\
\hline \multirow[t]{2}{*}{ Drogas } & No usa & 47 & 8,8 & 2 & $1,19-3,44$ & 5,68 & 0,01 \\
\hline & Si usa & 16 & 17,6 & & & & \\
\hline \multirow[t]{2}{*}{ Actividad Física } & No realiza & 20 & 9,3 & 1,14 & $0,68-1,92$ & 0,26 & 0,6 \\
\hline & Si realiza & 43 & 10,6 & & & & \\
\hline \multirow[t]{2}{*}{ Horas de sueño } & Igual o mayor a 7H & 12 & 9,4 & 1,09 & $0,59-2,0$ & 0,07 & 0,7 \\
\hline & Menor a 7H & 51 & 10,3 & & & & \\
\hline
\end{tabular}

c El denominador para el porcentaje corresponde a la variable de la fila RP Razón de Prevalencia

IC95\% Intervalo de Confianza del 95\%

RV Razón de Verosimilitud

Fuente: Estudio “Factores asociados al rendimiento académico en estudiantes universitarios en tiempos de Covid-19”

\section{Discusión}

El SARS COV 2 implica una serie de cambios y consecuencias que afectan en la salud a toda la humanidad, los estudiantes universitarios constituyen uno de los grupos más afectados durante esta pandemia, se encuentran alteraciones en su calidad de vida, relacionada con las emociones, cogniciones y conductas, hábitos poco saludables, sedentarismo, siendo estos índices de riesgo de enfermedad (12), además refiere que el estilo de vida se ve alterado por cambios de clase social, cultural, económico y mental, que pueden presentarse durante el proceso de formación académica, convirtiéndose en un importante factor de riesgo en el proceso salud -enfermedad, reflejándose en el estado de salud y el rendimiento académico.

En este estudio se evidenció un 10,1\% de estudiantes con bajo rendimiento académico, de los cuales el 28,6\% tuvieron sobrepeso, con una probabilidad del $63 \%$ de tener bajo rendimiento académico; el $82,5 \%$ de los estudiantes consumen comida chatarra, existiendo 3 veces mayor riesgo de presentar bajo rendimiento académico, el sexo masculino tiene 1,6 veces mayor probabilidad de obtener bajo rendimiento frente al sexo femenino. Un $85,7 \%$ de estudiantes consumen alcohol, $y$ el $25,4 \%$ consumen drogas, existiendo 2 
veces más probabilidad de presentar bajo rendimiento académico.

El asilamiento durante la pandemia puede afectar la salud mental de las personas, la aplicación del test de Zung ha demostrado ser inversamente proporcional al rendimiento académico, este puede deberse a que la depresión abarca actitudes negativas como la perdida de interés en las actividades cotidianas, alteraciones en la concentración y atención lo que conlleva a un descuido a las actividades destinadas al estudio (13).

El análisis bivariado entre IMC y rendimiento académico, demostró una relación inversamente proporcional entre las variables estudiadas, mientras más elevado el IMC en los estudiantes, el rendimiento académico será inferior, esto puede deberse a que las personas con sobrepeso tienen dificultad para conciliar el sueño, lo cual conlleva a dificultades en la concentración y la atención, así como también el consumo elevado de grasas y azúcares afecta el proceso de aprendizaje como se demuestra en las investigaciones de México y España (14-16).

Contrario de los hallazgos encontrados es esta investigación, en un estudio realizado en 156 estudiantes mexicanos de secundaria, en el cual se comparó el IMC con rendimiento académico, se demostró que el rendimiento académico alto fue más frecuente en el $40.9 \%$ de los estudiantes con Obesidad, $36,4 \%$ en peso normal, y 22,7\% en sobrepeso (17).

En los alumnos de una escuela de Australia, se encontró que los estudiantes con un alto consumo de azucares presentaron dificultades en su desempeño académico en distintas áreas evaluadas como lectura, escritura gramática, aritmética, de la misma forma se ha demostrado en varias investigaciones que el consumo de comida chatarra (comida rápida, procesada, alta en grasas) está muy relacionada con el rendimiento académico deficiente (18-20), sin embargo, existe evidencia en estudios con estudiantes escolares y de secundaria, lo que deja de lado a la población universitaria, por ello la importancia de esta investigación.

La calidad de la dieta está estrechamente relacionada con el rendimiento académico, los estudiantes con una inadecuada calidad de dieta, fueron más propensos a presentar un desempeño académico regular, mientras que aquello que venían de familias con un nivel socio-económico adecuado lo cual se reflejó directamente relacionado con una calidad de dieta adecuada, presentaban una mejor puntuación académica, lo cual se evidenció también en Chile en una población de 2116 estudiantes $(21,22)$.

Por otra parte, un estudio realizado en la Universidad Santiago de Guayaquil, con una muestra de 137 estudiantes destaca la falta de actividad física, consumo deficiente de agua, consumo de alimentos con escaso valor nutricional. El $64 \%$ de estudiantes presentaban consumo excesivo de alimentos altos en azucares, sal, carbohidratos y grasas saturadas, con riesgo a mediano y largo plazo de desencadenar enfermedades crónicas no transmisibles, contribuyendo negativamenteen el cuidado de la salud, obesidad, sedentarismo y afección del rendimiento académico (23). 
De igual manera, un estudio llevado a cabo en una escuela de la provincia del Guayas teniendo como muestra a 114 individuos donde se determinó la influencia de los hábitos alimenticios en el rendimiento escolar, obteniendo como conclusión que los hábitos alimenticios inadecuados influyen en las actividades del alumno y por ende en el rendimiento educativo del mismo obteniendo notas deficientes (24).

La actual pandemia puede repercutir negativamente en la salud mental de las personas y en sus hábitos de vida, existiendo una estrecha relación entre síntomas de trastornos depresivos y estrés, con pérdida de trabajo, insomnio, consumo de sustancias psicoactivas, bajo rendimiento académico, inadecuada percepción del estado de salud (25). De igual manera en un estudio llevado a cabo en la población colombiana sobre los hábitos de consumo durante el periodo de aislamiento obligatorio concluyeron que, la población estudiada entre los 18 a 30 años fue propensa a grandes cambios alimenticios al no tener un balance alimenticio óptimo, principalmente por problemas económicos y trastornos psicológicos que propician el desbalance nutricional (26).

Los factores asociados al consumo de sustancias psicoactivas en estudiantes universitarios son: el sexo masculino, depresión, mala relación familiar, bajo rendimiento académico, disfunción familiar $(27,28)$.

Iguales resultados se encontraron en un estudio realizado en 738 estudiantes de educación secundaria obligatoria, en el cual el $65 \%$ de los estudiantes consumía alcohol, el $25 \%$ declaro consumir cánnabis, registrándose una estrecha relación entre el consumo de estas sustancias con el rendimiento académico bajo y absentismo escolar (29).

\section{CONCLUSIONES}

En base a los resultados obtenidos se concluye que la mayor proporción de estudiantes de la carrera de Medicina durante el periodo académico octubre 2020 marzo 2021 cuyas clases se llevaron a cabo de forma virtual por la actual pandemia, adquirió hábitos como consumo de comida chatarra (hot dogs, salchipapas, pollo brosterizado, hamburguesas), bebidas energizantes, gaseosas, alcohólicas, drogas lo cual aumentó significativamente su bajo rendimiento, además de que el sexo masculino fue más propenso a tener bajo rendimiento durante el aislamiento, a pesar de los malos hábitos adquiridos por los estudiantes cabe recalcar que un gran porcentaje adquirió el hábito de realizar actividad física, mismos que manifestaron tener conciencia de que sus hábitos no son saludables, frente a esto es necesario promover hábitos de vida saludables para no solo mejorar el rendimiento académico de los estudiantes sino también, prevenir enfermedades no transmisibles a futuro.

Debido a los cambios en el estilo de vida que surgieron a partir de las medidas tomadas para la prevención de COVID-19, los estudiantes adquirieron nuevos hábitos de consumo y estilos de vida que comprometen 
ya sea a corto, mediano o largo plazo su rendimiento, lo que genera que los estudiantes pierdan su ciclo académico.

\section{REFERENCIAS BIBLIOGRÁFICAS}

1. Palacios Cruz $M$, Santos $E$, Velázquez Cervantes MA, León Juárez M. COVID-19, una emergencia de salud pública mundial. Rev Clin Esp. enero de 2021;221(1):55-61.

2. Fresno Ca del. Tasa de letalidad de la COVID-19 [Internet]. Empíreo Diagnóstico Molecular - Diagnóstico de VIH y ETS. 2021 [citado 21 de octubre de 2021]. Disponible en: https://www.empireo.es/tasa-de-letalidad-dela-covid-19/

3. Vera-Ponce VJ, Torres-Malca JR, TelloQuispe EK, Orihuela-Manrique EJ, Cruz-Vargas JADL, Vera-Ponce VJ, et al. Validación de escala de cambios en los estilos de vida durante el periodo de cuarentena en una población de estudiantes universitarios de Lima, Perú. Revista de la Facultad de Medicina Humana. octubre de 2020;20(4):614-23.

4. Sánchez-Ojeda MA, Luna-Bertos ED. Hábitos de vida saludable en la población universitaria. Nutrición Hospitalaria. mayo de 2015;31(5):1910-9.

5. Quichimbo YEP, Angulo PIU, Solorzano KGS, Villaruel KSO, Godoy MAG. Malos hábitos alimenticios y cansancio mental en los estudiantes universitarios del Ecuador (caso cantón Milagro). Anatomía Digital. 2019;2(4):60-70.

6. López Gutiérrez PP, Rejón Orantes J del C, Escobar Castillejos D, Roblero Ochoa SR, Dávila Esquivel MT, Mandujano Trujillo ZP. Conocimientos nutricionales en estudiantes universitarios del sector público del Estado de Chiapas, México. Investigación en Educación Médica. 1 de octubre de 2017;6(24):228-33.

7. Beltrán Corredor M. Estilo de vida en jóvenes universitarios de la facultad de psicología de la Fundación Universitaria de los Libertadores. reponame: Repositorio Institucional FULL [Internet]. 9 de diciembre de 2016 [citado
21 de octubre de 2021]; Disponible en: https://repository.libertadores.edu.co/ handle/11371/958

8. Guerra Cáceres EM. Hábitos alimenticios y su relación con el rendimiento académico de los estudiantes de la carrera profesional de Técnica en Farmacia del Instituto de Educación Superior Tecnológico Privado Cayetano Heredia, Arequipa. 2015. Universidad Nacional de San Agustín de Arequipa [Internet]. 2017 [citado 21 de octubre de 2021]; Disponible en: http://repositorio.unsa.edu.pe/handle/ UNSA/6583

9. González Cazorla N. Estado nutricional y su influencia en el rendimiento académico. $1 .^{a}$ ed. Madrid: Editorial Inclusión; 2021. 46 p.

10. Ramírez J, Castro-Quintero D, LermaCórdoba C, Yela J, Escobar-Córdoba F. CONSECUENCIAS DE LA PANDEMIA COVID 19 EN LA SALUD MENTAL ASOCIADAS AL AISLAMIENTO SOCIAL. Revista Colombiana de Anestesiologia. 3 de mayo de 2020;49(4):8.

11. Pfefferbaum B, North CS. Mental Health and the Covid-19 Pandemic. New England Journal of Medicine. 6 de agosto de 2020;383(6):510-2. 12. Veramendi Villavicencios NG, Portocarero Merino E, Espinoza Ramos FE, Veramendi Villavicencios NG, Portocarero Merino E, Espinoza Ramos FE. Estilos de vida y calidad de vida en estudiantes universitarios en tiempo de Covid-19. Revista Universidad y Sociedad. diciembre de 2020;12(6):246-51.

13. Diaz Vera DA. Asociación entre depresión y obesidad con el rendimiento académico en la IE FAP Samuel Ordoñez Velázquez Piura 2020. Universidad Privada Antenor Orrego [Internet]. 2021 [citado 25 de septiembre de 2021]; Disponible en: https://repositorio.upao. edu.pe/handle/20.500.12759/7718

14. Salazar-Rendón JC, Méndez N, Azcorra $\mathrm{H}$, Salazar-Rendón JC, Méndez N, Azcorra $\mathrm{H}$. Asociación entre el sobrepeso y la obesidad con el rendimiento académico en estudiantes de secundaria de la ciudad de Mérida, México. Boletín médico del Hospital Infantil de México. abril de 2018;75(2):94-103. 
15. Iglesias Á, Pozo EMP del, López JM. Prevalencia de sobrepeso y obesidad, hábitos alimentarios y actividad física y su relación sobre el rendimiento académico. Retos: nuevas tendencias en educación física, deporte y recreación. 2019;(36):167-73.

16. Peña García GM, Flores Leal KG. Habitos alimenticios y rendimiento escolar en jovenes de bachillerato. Caribeña de Ciencias Sociales [Internet]. 17 de enero de 2019 [citado 13 de octubre de 2021];(enero). Disponible en: https://www.eumed.net/rev/caribe/2019/01/ habitos-alimenticios-bachillerato.html

17. González MÁ, Juárez CLV, Rodríguez BAC, Picazo GD, Colunga JCV, Corona DMH, et al. Estudio transversal para comparar el rendimiento académico en estudiantes mexicanos de educación secundaria en función de su índice de masa corporal. Revista española de nutrición humana y dietética. 2020;24(3):218-25.

18. Burrows T, Goldman S, Olson RK, Byrne B, Coventry WL. Associations between selected dietary behaviours and academic achievement: A study of Australian school aged children. Appetite. 1 de septiembre de 2017;116:372-80.

19. Basilio Amado SE. Influencia del consumo de la comida chatarra en el rendimiento académico de los estudiantes del primer año del nivel secundario de la institución educativa Luis Fabio Xammar Jurado. Repositorio Institucional - UNJFSC [Internet]. 2015 [citado 13 de octubre de 2021]; Disponible en: http:// repositorio.unjfsc.edu.pe/handle/UNJFSC/521

20. López C, Fernanda M. La comida chatarra y su influencia en el rendimiento de los adolescentes. 2016 [citado 13 de octubre de 2021]; Disponible en: http://repositorio.ute. edu.ec/xmlui/handle/123456789/15599

21. Muñoz Villanueva $P$, Alarcón Araneda $M$, Jarpa-Parra $M$, Morales Ojeda IA, Muñoz Villanueva $P$, Alarcón Araneda $M$, et al. Hábito y calidad del desayuno; su efecto en el rendimiento académico de estudiantes de técnico de nivel superior en enfermería. Horizonte sanitario. diciembre de 2020;19(3):405-14.
22. Ibarra Mora J, Hernández Mosqueira CM, Ventura-Vall-Llovera C, Ibarra Mora J, Hernández Mosqueira CM, Ventura-VallLlovera C. Hábitos alimentarios y rendimiento académico en escolares adolescentes de Chile. Revista Española de Nutrición Humana y Dietética. diciembre de 2019;23(4):292-301.

23. Dávila Romero AL, Yánez Yar AN. Hábitos alimentarios de los estudiantes de la carrera de odontología y enfermería de la UCSG en el periodo de mayor confinamiento por Covid-19. 17 de septiembre de 2020 [citado 26 de septiembre de 2021]; Disponible en: http:// repositorio.ucsg.edu.ec/handle/3317/15235

24. Quimis Tumbaco MM, Vallejo Gonzalez GM. La nutrición y su influencia en el rendimiento escolar de los estudiantes de octavo grado de Educación General Básica. Diseño de una campaña informativa con enfoque a la nutrición [Internet]. 2019 [citado 13 de octubre de 2021]; Disponible en: http://repositorio. ug.edu.ec/handle/redug/40260

25. Gómez JAM, Suárez YB, Yanez-Peñúñuri LY, Anacona CAR. Tendencias de la investigación sobre síntomas de trastornos mentales durante la pandemia por COVID-19. Medicina UPB. 2020;39(2):24-33.

26. Barker Archbold SS, Díaz Ríos AN, Osorio Rodríguez ND. Impacto del cambio en los hábitos alimenticios sobre la salud de la población entre los 18 y 30 años por el confinamiento obligatorio en Colombia. 21 de enero de 2021 [citado 13 de octubre de 2021]; Disponible en: https://repository.ean.edu.co/ handle/10882/10266

27. Taboada Villarreyes VF. Factores asociados al consumo de sustancias psicoactivas en estudiantes de medicina de la Universidad Nacional de Piura, en el contexto de la pandemia por Covid-19, durante el primer semestre del 2021. Universidad Nacional de Piura [Internet]. 2021 [citado 13 de septiembre de 2021]; Disponible en: http://repositorio.unp. edu.pe/handle/20.500.12676/2770

28. Montero AP, Perez JC, Serrano LJ, Cansino SR. El Consumo de drogas y su incidencia en 
el Nivel de Rendimiento Académico en los estudiantes de los Octavos Años de Educación General Básica de la Ciudad de Quito. Práctica Familiar Rural [Internet]. 29 de marzo de 2019 [citado 13 de octubre de 2021];4(1). Disponible en: $\quad$ https://practicafamiliarrural.org/index. $\mathrm{php} / \mathrm{pfr} / \mathrm{article} / \mathrm{view} / 40$

29. Navalon-Mira A, Ruiz-Callado R. Consumo de sustancias psicoactivas y rendimiento académico. Una investigación en estudiantes de educación secundaria obligatoria. Substance use associated with academic performance $A$ research about secondary education students [Internet]. 2017 [citado 26 de septiembre de 2021]; Disponible en: http://rua.ua.es/dspace/ handle/10045/62547

\section{ACERCA DE LOS AUTORES}

Sara Elizabeth Bravo Salinas. Médico General, Universidad Católica de Cuenca. Médica Especialista en Medicina Familiar, Universidad Central del Ecuador. Máster en Atención de pacientes con VIH, Universidad Rey Juan Carlos de España. Especialista en Docencia Universitaria, Universidad del Azuay. Docente Universidad Tecnológica Equinoccial, Universidad Católica de Cuenca, Ecuador.

Alex Alberto Castillo Zhizhpón. Médico General, Universidad Católica de Cuenca. Médico Especialista en Medicina Familiar, Universidad Central del Ecuador. Máster en Atención de pacientes con VIH, Universidad Rey Juan Carlos de España Médico tratante del IESS. Universidad Católica de Cuenca, Ecuador.

Diana Carolina Izquierdo Coronel. Médico General, Universidad Estatal de Cuenca. Médica Especialista en Nutrición con Orientación en Obesidad, Universidad Favaloro Buenos Aires Argentina. Master en Diabetología, Universidad Del Salvador Buenos Aires Argentina. Posgrado en Diabetes Universidad Católica Buenos Aires Argentina. Diplomado de Soporte Metabólico Nutricional. Médico tratante Hospital Universitario del Río Docente de la Universidad Católica de Cuenca, Ecuador.

Paola Andrea Rodas Bermeo. Médico General graduado, Universidad Católica de Cuenca. Médico Asistencial de Medicina Interna, Hospital Homero Castanier Crespo. Médico de consulta externa del IESS. Cursando Especialidad en Docencia Universitaria. Universidad Católica de Cuenca, Ecuador.
Conflicto de intereses. El presente estudio se desarrolló bajo la aprobación de la Universidad Católica de Cuenca, previa autorización del departamento de Investigación, luego de ganar el concurso de Proyectos por Covid-19, manteniendo los preceptos de la Declaración de Helsinki de la Asociación Mundial con la finalidad de mantener las consideraciones éticas, además no se requirió ayuda financiera de ninguna institución gubernamental o no gubernamental, los autores declaran no tener ningún conflicto de intereses.

Agradecimiento. A la Universidad Católica de Cuenca sede Azogues, sus estudiantes y a cada uno de los participantes que hicieron posible dicha publicación.

Financiación: Ninguna declarada por los autores. 